\title{
THEORY OF EXACT SOLUTIONS FOR THE EVOLUTION OF A FLUID ANNULUS IN A ROTATING HELE-SHAW CELL
}

\author{
BY \\ DARREN CROWDY \\ Department of Mathematics, Imperial College of Science, Technology and Medicine, \\ 180 Queen's Gate, London
}

\begin{abstract}
Motivated by a series of recent experiments on the evolution of fluid annuli in rotating Hele-Shaw cells, this paper presents a new class of exact time-dependent solutions to a mathematical model of this nonlinear free boundary problem. For a certain class of initial conditions, the free boundary problem is reduced to the solution of a finite set of coupled nonlinear ordinary differential equations. These solutions can be explicitly studied and, despite the fact that the model problem is mathematically ill-posed, display the same qualitative features as the recent experiments. It is discussed how the present exact solutions might form an important basis for further study of the appropriately regularized model problem.
\end{abstract}

1. Introduction. There has been much recent interest in the morphological instabilities of interfaces in rotating Hele-Shaw cells. The problem has been studied numerically, experimentally, and theoretically over the last few years. Schwartz [17] examined the problem numerically (using boundary integral techniques) and studied the linear stability and nonlinear evolution of a liquid mass in a Hele-Shaw cell rotating at a constant angular velocity. Laboratory experiments examining the instability of an interface (of a dense fluid such as oil injected into air) driven by a centrifugal force in a rotating Hele-Shaw cell were presented by Carrillo et al. [2] with further experiments performed much more recently for the case of a fluid annulus in a rotating cell [3], [4]. In addition, theoretical developments have been made by Entov et al. [11] who used the Cauchy transform approach. Entov et al. [11] produce some exact time-dependent solutions for the free surface evolution of simply-connected blobs of fluid in a rotating cell. The dynamical solutions are described by rational function conformal maps from the unit circle in a parametric conformal mapping plane.

Received June 22, 1999.

2000 Mathematics Subject Classification. Primary 30-XX, 31-XX, 35-XX.

Key words and phrases. Nonlinear free boundary problem; fluid dynamics; rotating flows; Hele-Shaw.

E-mail address: d.crowdy@ic.ac.uk 
The evolution of an annular blob, as studied in the recent experiments of Carrillo et al. [3], [4], is of greater interest because the interplay between two disjoint interfaces potentially gives rise to a now phenomenology not available in the simply-comnected case where just one interface is present. In particular, as discussed in [3]-[4], the problem of a fluid annulus in a rotating Hele-Shaw cell is a physical system simultaneously susceptible to two distinct types of morphological instability. The first is the well-known Saffman-Taylor instability which occurs when a less viscous fluid is driven into a more viscous fluid. As a fluid ammulus is flung out radially due to the centrifugal forces in a rotating cell, this situation occurs at the inner, trailing interface. This instability is a direct result of the difference in viscosity between the two fluids. However there is, in addition, a second morphological instability at the outer interface which is more directly induced by the centrifugal forcing that is driven by the density difference of the two fluids. In recent experiments. Carrillo et al. [4] have observed that, depending on the densities and viscosities of the fluids in different regions of the cell, as well as on the experimental parameters selected (e.g., gap width, initial volume of the fluid annulus, rate of rotation of the cell) qualitatively different scenarios of interfacial instabilities are observed. According to the various combinations of parameters, either the imner, the outer, or both interfaces simultancously are observed to become unstable. We refer the reader to the original study for more details [4].

It is clearly desirable to be able to study the interaction of these two different instabilities analytically. Carrillo et al. [4] have carried out a linear stability analysis of the simple time-evolving concentric annulus solution (in which the two interfaces are simpler concentric circles of different radii) and observed that the two interfaces are coupled through the pressure field at the linear level. From the point of view of a fully nonlinear theory, a glimmer of hope is provided by the existence of the exact solutions to the rotating Hele-Shaw problem found by Entov et al. [11]. However, these solutions deal only with simply-connected domains in which just one interface is present and so are of no direct use in analyzing the situation of interest here, i.e., the effect of two distinct competing instabilities simultaneously acting on two disjoint free surfaces bounding an annular fluid region. This is the essence of the problem of a fluid annulus in a rotating Hele-Shaw cell, and it is not captured by any presently-known analytical solutions.

In light of this, it is the purpose of this paper to appropriately generalize the solutions of Entov et al. to a doubly-connected fluid domain, in two dimensions, with a view to providing a theoretical basis for understanding some of the recent experimental observations [3], [4]. By consideration of the theory of quadrature domains, it is established herein that the problem of Hele-Shaw flows in a rotating cell (with no sources or sinks, although these can also be incorporated into the theory) is such that conformal maps (from a fundamental ammulus in a parametric $\zeta$-plane to the doubly-connected physical fluid domain) that are initially loxodromic functions (defined later) remain loxodromic functions under evolution. This turns out to provide an explicit class of time-dependent solutions for the problem of the evolution of the boundaries of a fluid annulus in a rotating cell which are "exact" in the sense that the evolution is governed by a finite set of time-evolving parameters. For a special (but dense) class of initial conditions, the free boundary problem is thereby reduced to the solution of a finite set of coupled nonlinear 
ordinary differential equations. Furthermore, the relevant equations of evolution can be written down explicitly, and a class of invariants of the motion identified.

2. Description of the physical problem. Consider a blob $D(t)$ of fluid in a HeleShaw cell which will be assumed to be rotating with a (possibly time-dependent) angular velocity $\omega(t)$. The fluid is assumed to be evolving as a two-dimensional flow in the plane. For the purposes of this paper, the fluid blob will either be simply-connected or doublyconnected. In a frame rotating with the cell (and under similar approximations of high Reynolds numbers and high Rossby numbers as assumed in Carrillo et al. [2], [3]) the usual Darcy's law for Hele-Shaw flow, modified to include centrifugal forces, takes the form

$$
\nabla p=-\frac{\mu}{k} \mathbf{u}+\tilde{\rho} \omega^{2}(t) r \mathbf{e}_{r},
$$

where $\tilde{\rho}$ is the fluid density, $\mu$ the viscosity, $p$ the fluid pressure, and $k=b^{2} / 12$, where $b$ is the plate spacing in the cell. $\mathbf{e}_{r}$ is a unit vector in the radial direction. The fluid is incompressible, so that

$$
\nabla \cdot \mathbf{u}=0
$$

while the pressure condition on interfaces 1 and 2 is given by

$$
p=p_{j}(t)
$$

for some purely time-evolving parameters $p_{1}(t), p_{2}(t)$. Physically, these correspond to the pressures inside and outside the blob. Without loss of generality, we can set $p_{1}(t)=0$. The kinematic condition is that

$$
\mathbf{u} \cdot \mathbf{n}=V_{n},
$$

where $V_{n}$ is the normal velocity of the interface. The centrifugal term in (1) is removed by defining

$$
p=\hat{p}+\frac{\tilde{\rho} \omega^{2}(t) r^{2}}{2}
$$

so that

$$
\nabla \hat{p}=-\frac{\mu}{k} \mathbf{u}
$$

Taking the divergence of this equation implies that

$$
\nabla^{2} \hat{p}=0
$$

everywhere in $D(t)$, while the pressure condition on the interfaces becomes

$$
\hat{p}=-\frac{\tilde{\rho} \omega^{2}(t) r^{2}}{2}+p_{j}(t) \quad \text { on } \partial D_{j}(t) .
$$

Once the pressure field $\hat{p}$ is instantaneously determined, the fluid velocity follows from

$$
\mathbf{u}=-\frac{k}{\mu} \nabla \hat{p}
$$


From the above, it is clear that the non-dimensionalized free boundary problem in a rotating Hele-Shaw cell is equivalent to the following problem for a velocity potential $\phi=-\frac{k}{\mu} \hat{p}$ :

$$
\nabla^{2} \phi=0
$$

everywhere in $D(t)$. On the boundaries,

$$
\phi=\frac{A(t)}{2} z \bar{z}+B_{j}(t) \text { on } \partial D_{j}(t),
$$

where $A(t)=\tilde{\rho} \omega^{2}(t) k / \mu>0$ and where $B_{j}(t)=k p_{j}(t) / \mu$ is related to the air pressures inside and outside the annular blob. We have already determined that $B_{1}(t) \equiv 0$. A kinematic condition on each interface also requires that the local normal velocity of the boundary $V_{n}$ equals the local normal velocity of the fluid, i.e.,

$$
\nabla \phi \cdot \mathbf{n}=V_{n} .
$$

Note that the kinematic boundary condition (12) can be rewritten as

$$
\operatorname{Im}\left[\frac{d z}{d t} \bar{z}_{s}\right]=\operatorname{Im}\left[(u+i v) \bar{z}_{s}\right]=\operatorname{Im}\left[2 \phi_{\bar{z}} \bar{z}_{s}\right] \quad \text { on } \partial D_{j}(t) .
$$

This completes the specification of the free boundary problem. Mathematically, for a given boundary shape $D(t)$, condition (11) instantaneously determines the harmonic function $\phi$ at each instant of time while the subsequent evolution of $D(t)$ is then determined by the kinematic condition (13).

3. Mathematical formulation. We now define the linear functional $\mathcal{L}$ acting on functions $h(z, t)$ analytic and single-valued everywhere in $D(t)$ and continuous on $\partial D(t)$ :

$$
\mathcal{L}[h(z, t) ; D(t)] \equiv \iint_{D(t)} h(z, t) d x d y .
$$

Note that the analysis of this section will refer to a fluid region $D(t)$. For present purposes, we assume that this region is at most doubly-connected. Note, however, that the analysis of this section in fact holds for any region of finite connectivity. The following definition is from Davis [10]:

Definition. The linear functional $\mathcal{L}$ will be said to be a point differential functional of finite order if it can be expressed in the following form:

$$
\mathcal{L}[h(z)]=\sum_{n=1}^{N} \sum_{k=0}^{n_{k}} a_{n k} h^{(k)}\left(\tilde{z}_{n}\right),
$$

where $\tilde{z}_{1}, \tilde{z}_{2}, \ldots, \tilde{z}_{N}$ are a finite set of distinct points in the interior of $D$, and the $a_{n k}$ are constants. The points $\left\{\tilde{z}_{j}\right\}$ and the constant $a_{n k}$ are independent of the function $h(z)$, as are the integers $\left\{n_{k} \geq 0\right\} . h^{(k)}$ refers to the $k$ th derivative of $h(z)$ with respect to its argument.

In order to prove Theorem 3.2, we will require the following standard theorem [16] from the theory of harmonic functions: 
THEOREM 3.1. If $\phi(x, y)$ is harmonic in any region $D(t)$ (not necessarily simply-connected), then the function $\phi_{z}$ is holomorphic in $D(t)$. In particular, for any function $\phi$ harmonic in $D(t)$ the partial derivatives $\phi_{x}$ and $\phi_{y}$ are always the real parts of functions holomorphic in $D(t)$.

Proof. See [16].

REMARK 1. Note that if $\phi$ is harmonic in $D(t)$ and if $D(t)$ is not simply-connected, then there need not exist in $D(t)$ a holomorphic function having $\phi$ as its real part. However, Theorem 3.1 shows that this is not true of $\phi_{z}$.

THEOREM 3.2. In the free boundary problem just described, the following expression holds for the time evolution of $\mathcal{L}[h(z, t) ; D(t)]$ :

$$
\frac{d}{d t} \mathcal{L}[h(z, t) ; D(t)]=\mathcal{L}\left[h_{t}(z, t)+A(t) z h_{z}(z, t) ; D(t)\right]
$$

where $h(z, t)$ is analytic and single-valued in $D(t)$ and continuous on $\partial D(t)$.

Proof. Using the complex form of Green's theorem in the plane,

$$
\mathcal{L}[h(z, t) ; D(t)]=\frac{1}{2 i} \oint_{D(t)} h(z, t) \bar{z} d z .
$$

We now compute the time derivative of this quantity:

$$
\frac{d \mathcal{L}[h(z, t) ; D(t)]}{d t}=\frac{1}{2 i} \oint_{\partial D(t)} h_{t} \bar{z} d z+h_{z} z_{t} \bar{z} d z+h\left(\bar{z}_{t} d z+\bar{z} d_{t}\right) .
$$

But from (13),

$$
\bar{z}_{t} d z=z_{t} d \bar{z}+2 \phi_{z} d z-2 \phi_{\bar{z}} d \bar{z}
$$

which, when substituted into (18), gives

$$
\begin{aligned}
\frac{d \mathcal{L}[h(z, t) ; D(t)]}{d t}= & \frac{1}{2 i} \oint_{\partial D(t)} h_{t} \bar{z} d z+h_{z} z_{t} \bar{z} d z+h \bar{z} d z_{t}+h z_{t} d \bar{z} \\
& +2 h\left(\phi_{z} d z-\phi_{\bar{z}} d \bar{z}\right) .
\end{aligned}
$$

But

$$
h_{z} z_{t} \bar{z} d z+h \bar{z} d z_{t}+h z_{t} d \bar{z}=d\left(z_{t} h(z) \bar{z}\right)
$$

is a total (spatial) differential and therefore gives zero contribution, while it is known from (11) that on $\partial D(t)$

$$
\phi_{\bar{z}} d \bar{z}=d\left(\frac{A(t)}{2} z \bar{z}\right)-\phi_{z} d z
$$

which, when substituted into (20), gives

$$
\frac{d \mathcal{L}[h(z, t) ; D(t)]}{d t}=\frac{1}{2 i} \oint_{\partial D(t)} h_{t} \bar{z} d z+2 h\left(2 \phi_{z} d z-d\left(\frac{A(t)}{2} z \bar{z}\right)\right) .
$$


But by Theorem $3.1,2 \phi_{z}$ is analytic everywhere in $D(t)$, which means that, regardless of the connectivity of $D(t)$, Cauchy's Theorem can be applied to one of the integrals in (23) and leaves, after an integration by parts,

$$
\frac{d \mathcal{L}[h(z, t): D(t)]}{d t}=\frac{1}{2 i} \oint_{\partial D(t)} h_{t} \bar{z} d z+A(t) z h_{z} \bar{z} d z,
$$

which, using Green's theorem, provides the required result.

We now show why this result implies that the dynamics is such as to preserve the finite order of an initial point differential functional of finite order.

Let $h(z, t)$ be a given function of $z$ and $t$. Given $h$, define a function $\hat{h}$ via the equation

$$
\hat{h}(\hat{z}(z, t), t)=h(z, t),
$$

which is assumed to hold at all times $t$, where $\hat{z}(z, t)$ is the solution of the complex partial differential equation

$$
\frac{\partial \hat{z}}{\partial t}+A(t) z \frac{\partial \hat{z}}{\partial z}=0 ; \quad \hat{z}(z, 0)=z .
$$

The above partial differential equation can be solved explicitly, and it is easy to see that the solution is given by

$$
\hat{z}(z, t)=z e^{-\int_{0}^{t} A\left(t^{\prime}\right) d t^{\prime}}
$$

It will be assumed that the function $h(z, t)$ is completely arbitrary except that it must be an analytic function of $z$ everywhere in $D(0) \cup D(t)$ for $0<t<T$ where $T$ is some nonzero radius of convergence defined later.

We first prove a uscful lemma:

Lemma. The following result is true:

$$
\left(\frac{\partial}{\partial t}+A(t) z \frac{\partial}{\partial z}\right)^{n} h(z, t)=\hat{h}^{(0 . n)}(\hat{z}(z, t), t)
$$

for $n \geq 1$ and where the notation $g^{(m, n)}(-,-)$ refers to the $m$ th derivative with respect to the first argument and the $n$th derivative with respect to the second argument.

Proof. The proof is by induction. Consider the case $n=1$ :

$$
\begin{aligned}
\left(\frac{\partial}{\partial t}+A(t) z \frac{\partial}{\partial z}\right) h(z, t) & =\left(\frac{\partial}{\partial t}+A(t) z \frac{\partial}{\partial z}\right) \hat{h}(\hat{z}(z, t), t) \\
& =\hat{h}^{(0,1)}(\hat{z}, t)+\hat{h}^{(1,0)}(\hat{z}, t)\left(\frac{\partial \hat{z}}{\partial t}+A(t) z \frac{\partial \hat{z}}{\partial z}\right) \\
& =\hat{h}^{(0,1)}(\hat{z}, t),
\end{aligned}
$$

where we have used (25) and (26). Now, suppose that the result is true for an arbitrary integer $n \geq 1$. Then

$$
\left(\frac{\partial}{\partial t}+A(t) z \frac{\partial}{\partial z}\right)^{n} h(z, t)=\hat{h}^{(0, n)}(\hat{z}, t)
$$


which implies that

$$
\begin{aligned}
\left(\frac{\partial}{\partial t}+A(t) z \frac{\partial}{\partial z}\right)^{n+1} h(z, t) & =\left(\frac{\partial}{\partial t}+A(t) z \frac{\partial}{\partial z}\right) \hat{h}^{(0, n)}(\hat{z}(z, t), t) \\
& =\hat{h}^{(1, n)}\left(\frac{\partial}{\partial t}+A(t) z \frac{\partial}{\partial z}\right) \hat{z}(z, t)+\hat{h}^{(0, n+1)}(\hat{z}, t) \\
& =\hat{h}^{(0, n+1)}(\hat{z}, t)
\end{aligned}
$$

by (26). The result follows for all integers by induction.

Theorem 3.3. Assuming the boundary evolution is locally analytic in time, the following expression holds for times $0<t<T$ where $T$ is some nonzero radius of convergence:

$$
\mathcal{L}[h(z, t) ; D(t)]=\mathcal{L}\left[h\left(z e^{\int_{0}^{t} A\left(t^{\prime}\right) d t^{\prime}}, t\right) ; D(0)\right] .
$$

Proof. Assuming that the boundary evolution is locally analytic in time, this integral can be Taylor expanded about $t=0$ for $t$ within some radius of convergence $T$, i.e.,

$$
\begin{aligned}
\iint_{D(t)} h(z, t) d x d y= & \left.\iint_{D(t)} h(z, t) d x d y\right|_{t=0}+\left.t \frac{d}{d t} \iint_{D(t)} h(z, t) d x d y\right|_{t=0} \\
& +\left.\frac{t^{2}}{2 !} \frac{d^{2}}{d t^{2}} \iint_{D(t)} h(z, t) d x d y\right|_{t=0}+\cdots .
\end{aligned}
$$

These derivatives can be computed by repeatedly using Theorem 3.2 and the lemma above,

$$
\begin{aligned}
\iint_{D(t)} h(z, t) d x d y= & \iint_{D(0)}\left(\hat{h}(z, 0)+t \hat{h}^{(0,1)}(z, 0)\right. \\
& \left.+\frac{t^{2}}{2 !} \hat{h}^{(0,2)}(z, 0)+\cdots\right) d x d y \\
& =\iint_{D(0)} \hat{h}(z, t) d x d y
\end{aligned}
$$

after resumming the Taylor series expansion for $\hat{h}(z, t)$ about $t=0$. But since

$$
\hat{z}(z, t)=z e^{-\int_{0}^{t} A\left(t^{\prime}\right) d t^{\prime}},
$$

then

$$
\hat{z}\left(z e^{\int_{0}^{t} A\left(t^{\prime}\right) d t^{\prime}}, t\right)=z .
$$

Substituting this expression for $z$ into the right-hand side of (34) implies

$$
\begin{aligned}
\iint_{D(t)} h(z, t) d x d y & =\iint_{D(0)} \hat{h}\left(\hat{z}\left(z e^{\int_{0}^{t} A\left(t^{\prime}\right) d t^{\prime}}, t\right), t\right) d x d y \\
& =\iint_{D(0)} h\left(z e^{\int_{0}^{t} A\left(t^{\prime}\right) d t^{\prime}}, t\right) d x d y
\end{aligned}
$$

the last equation following from the definition (25). 
THEOREM 3.4. If the initial domain $D(0)$ is such that $\mathcal{L}[h(z) ; D(0)]$ is a point differential functional of finite order, i.e..

$$
\iint_{D(0)} h(z) d x d y=\left.\sum_{n=1}^{N} \sum_{k=0}^{n_{k}} a_{n k} \frac{\partial^{k}}{\partial z^{k}} h(z)\right|_{\tilde{z}_{n}}
$$

for some $\left\{a_{n k}\right\}$ and $\left\{\tilde{z}_{n}\right\}$ (the points $\left\{\tilde{z}_{n}\right\}$ assumed strictly inside $D(0)$ ) and arbitrary $h(z)$ analytic in $D(0)$, then for sufficiently short times $0<t<T, \mathcal{L}[h(z, t) ; D(t)]$ remains a point differential functional of finite order.

Proof. Using (32) and the fact that $\mathcal{L}[h(z) ; D(0)]$ is a point differential functional of finite order, we obtain

$$
\begin{aligned}
\iint_{D(t)} h(z, t) d x d y & =\iint_{D(0)} h\left(z e^{\int_{0}^{t} A\left(t^{\prime}\right) d t^{\prime}}, t\right) d x d y \\
& =\left.\sum_{n=1}^{N} \sum_{k=0}^{n_{k}} a_{n k} \frac{\partial^{k} h\left(z e^{\int_{0}^{t} A\left(t^{\prime}\right) d t^{\prime}}, t\right)}{\partial z^{k}}\right|_{\tilde{z}_{n}}
\end{aligned}
$$

(assuming that sufficiently short times have elapsed that $h\left(z e^{\int_{0}^{t} A\left(t^{\prime}\right) d t^{\prime}}, t\right)$ remains analytic in $D(0)$ ). But it is clear (by the chain rule) that this expression has the general form

$$
\iint_{D(t)} h(z, t) d x d y=\sum_{n=1}^{N} \sum_{k=0}^{n_{k}} d_{n k}(t) h^{(k, 0)}\left(z_{n}(t), t\right),
$$

where the time-dependent coefficient $d_{n k}(t)$ can be found explicitly if required and $d_{n k}(0)=a_{n k}$. The points $z_{j}(t)$ are defined as follows:

$$
z_{n}(t)=\tilde{z}_{n} e^{\int_{0}^{t} A\left(t^{\prime}\right) d t^{\prime}}, \quad n=1, \ldots, N .
$$

Thus, at least for sufficiently short times, $\mathcal{L}[h(z, t) ; D(t)]$ is a point differential functional of finite order (defined at the time-evolving points $z_{j}(t)$ ). The theorem is then proved.

REMARK 2 (Invariants). From (39) and (40), it can be seen, by considering $k=0$ in the sum, that the coefficients $d_{n 0}(t)$ of $h\left(z_{j}(t), t\right)$ at any later time $t$ are just $a_{n 0}$, i.e., the same as the initial values. This means that $d_{n 0}(t)=a_{n 0}$ are invariants of the motion. The existence of these invariants of the motion does not seem to have been explicitly pointed out before, even in the simply-connected case.

REMARK 3. The foregoing proof has many similarities with that presented in [6] in the context of the quite distinct problem of viscous sintering. Indeed, Crowdy [8] has shown that the rotating Hele-Shaw problem and the viscous sintering problem have many surprising, but important, mathematical similarities. Indeed, these similarities have led the present author to the new exact solutions for the evolution of the fluid annulus to be presented herein.

REMARK 4. Domains over which there exists a point differential functional of the form (40) are also referred to as quadrature domains. 
4. Evolution equations for the mapping. The analysis of the previous section holds regardless of the connectivity of the fluid domain. In this section, we separately consider the cases of simply-connected and doubly-connected fluid regions. While the present mathematical approach is different, the following exact solutions for simplyconnected regions have already been identified by previous investigators using the mathematical notion of a Cauchy transform of a domain [11], [8]. However, the following exact loxodromic function solutions, which we derive for a doubly-connected fluid annulus rotating in a Hele-Shaw cell, are new. Moreover, there are some additional nontrivial mathematical complications involved in calculating this latter class of solutions, which do not arise in the simply-connected case.

4.1. Simply-connected case.

Corollary 4.1. If the conformal map $z(\zeta, 0)$ from the unit $\zeta$-circle to the initial blob $D(0)$ satisfying $z(0,0)=0$ is a rational function, then it remains a rational function for (sufficiently short) times $t>0$.

Proof. The proof of this result follows from Theorem 3.4, which shows that $\mathcal{L}[h(z, t)$; $D(t)$ ] remains a point differential functional (of finite order) if it is initially, combined with the fact that the conformal map (satisfying $z(0, t)=0$ ) from the unit $\zeta$-circle to a domain $D(t)$ on which $\mathcal{L}$ is a point differential functional is necessarily a rational function. The proof of this latter result can be found in Davis [10].

EXAmple 4.1.1. Consider a point differential functional of finite order given as

$$
\mathcal{L}[h(z, t) ; D(t)]=a_{1}(t) h\left(z_{1}(t), t\right)+a_{2}(t) h\left(z_{2}(t), t\right) .
$$

Note that since, by Theorem 3.4 , the linear functional $\mathcal{L}$ remains a point differential functional of finite order (having the form (42)) under evolution, then there are four parameters $\left\{z_{1}(t), z_{2}(t), a_{1}(t), a_{2}(t)\right\}$ determining the evolution of $D(t)$. Differentiating (42) directly with respect to time yields

$$
\begin{aligned}
\frac{d \mathcal{L}[h(z, t) ; D(t)]}{d t}= & \dot{a}_{1}(t) h\left(z_{1}(t), t\right)+a_{1}(t) \dot{z}_{1} h^{(1,0)}\left(z_{1}(t), t\right)+a_{1}(t) h^{(0,1)}\left(z_{1}(t), t\right) \\
& +\dot{a}_{2}(t) h\left(z_{2}(t), t\right)+a_{2}(t) \dot{z}_{2} h^{(1,0)}\left(z_{2}(t), t\right)+a_{2}(t) h^{(0,1)}\left(z_{2}(t), t\right) .
\end{aligned}
$$

But it is also true that

$$
\frac{d \mathcal{L}[h(z, t) ; D(t)]}{d t}=\mathcal{L}\left[h_{t}(z, t)+A(t) z h_{z}(z, t) ; D(t)\right]
$$

and the right-hand side of (44) is a point differential functional of finite order, so that

$$
\begin{aligned}
\frac{d \mathcal{L}[h(z, t) ; D(t)]}{d t}= & \mathcal{L}\left[h_{t}(z, t)+A(t) z h_{z}(z, t) ; D(t)\right] \\
= & a_{1}(t)\left(h^{(0,1)}\left(z_{1}(t), t\right)+A(t) z_{1}(t) h^{(1,0)}\left(z_{1}(t), t\right)\right) \\
& +a_{2}(t)\left(h^{(0,1)}\left(z_{2}(t), t\right)+A(t) z_{2}(t) h^{(1,0)}\left(z_{2}(t), t\right)\right) .
\end{aligned}
$$

Inspection of (43) and (45) reveals that they can only be consistent for all choices of $h(z, t)$ provided that

$$
\begin{array}{r}
\dot{a}_{j}(t)=0, \quad j=1,2, \\
\dot{z}_{j}(t)=A(t) z_{j}(t), \quad j=1,2 .
\end{array}
$$


The parameters $z_{1}(t), z_{2}(t), a_{1}(t)$, and $a_{2}(t)$ are therefore explicitly given by

$$
\begin{aligned}
& z_{j}(t)=z_{j}(0) e_{0}^{\int_{0}^{t} A\left(t^{\prime}\right) d t^{\prime}}, \quad j=1,2, \\
& a_{j}(t)=a_{j}(0), \quad j=1,2 .
\end{aligned}
$$

Note the existence of two invariants of the motion. It now remains to determine the corresponding conformal map. It is clear that these must be derivable in some way from knowledge (at each instant) of $a_{1}(t), a_{2}(t), z_{1}(t)$, and $z_{2}(t)$. These latter quantities are known from (47).

Now restrict to the case of steady rotation, and pick $A=1$. Consider initial conditions in which

$$
a_{2}(0)=a_{1}(0), \quad z_{2}(0)=-z_{1}(0),
$$

where all quantities are assumed real. These initial conditions correspond to an example presented in [11]. From (47), consistent solutions of the dynamical equations are given by

$$
a_{1}(t)=a_{2}(t), \quad z_{2}(t)=-z_{1}(t)
$$

for all times $t>0$ for which a solution exists. The conformal map from the unit $\zeta$-circle to such a quadrature domain can be deduced to be given by

$$
z(\zeta, t)=\frac{R(t) \zeta}{\zeta^{2}-p(t)^{2}}
$$

where $R(t)$ and $p(t)$ are real. This is readily verified by use of the complex form of Green's theorem and the residue theorem. Indeed, it is straightforward to show the following correspondence between the parameters $p(t)$ and $R(t)$ in the conformal map and the parameters $z_{1}(t), a_{1}(t)$ in the quadrature identity

$$
\begin{aligned}
z_{1}(t) & =\frac{R(t) p(t)}{1-p(t)^{4}}, \\
a_{1}(t) & =\frac{\pi R(t)^{2}}{2} \frac{\left(1+p(t)^{4}\right)}{\left(1-p(t)^{4}\right)^{2}} .
\end{aligned}
$$

The evolution equations for $p(t)$ and $R(t)$ are therefore given implicitly by the equations

$$
\begin{aligned}
& z_{1}(t)=z_{1}(0) e^{t}=z\left(p(t)^{-1}, t\right), \\
& a_{1}(t)=a_{1}(0)=\pi \operatorname{Residue}\left[\bar{z}\left(\zeta^{-1}, t\right) z_{\zeta}(\zeta, t) ; p(t)^{-1}\right] .
\end{aligned}
$$

4.2. Doubly-connected case. To treat the doubly-connected case, it is necessary to introduce a function $P(\zeta ; \rho)$ defined by the infinite product

$$
P(\zeta ; \rho) \equiv(1-\zeta) \prod_{k=1}^{\infty}\left(1-\rho^{2 k} \zeta\right)\left(1-\rho^{2 k} \zeta^{-1}\right) .
$$

This function satisfies the following functional equations:

$$
\begin{gathered}
P\left(\zeta^{-1} ; \rho\right)=-\zeta^{-1} P(\zeta ; \rho), \\
P\left(\rho^{2} \zeta ; \rho\right)=-\zeta^{-1} P(\zeta ; \rho) .
\end{gathered}
$$

By Riemann's Theorem for a doubly-connected fluid region, at each instant of time, there exists a mapping $z(\zeta, t)$ from the ammulus $C_{0}$ (defined as the annulus $\rho(t)<|\zeta|<1$ ) 
for some $\rho(t)$ which conformally maps to the physical fluid region. The conformal modulus $\rho(t)$ must be found as part of the solution. (See Fig. 0.)

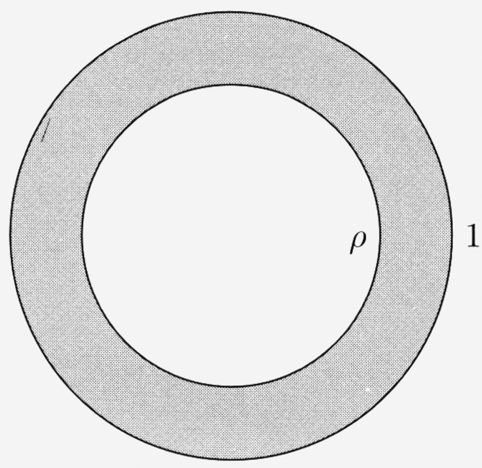

FIG. 0. Parametric $\zeta$-plane

Consider the special class of conformal mappings from the annulus $\rho(t)<|\zeta|<1$ to a fluid annulus in the plane having the functional form

$$
z(\zeta, t)=R(t) \zeta \frac{\prod_{k=1}^{N} P\left(\zeta \eta_{k}(t)^{-1} ; \rho(t)\right)}{\prod_{k=1}^{N} P\left(\zeta \zeta_{k}(t)^{-1} ; \rho(t)\right)},
$$

where $N$ is some integer $(N \geq 2)$ and the parameters $\left\{\eta_{j}(t)\right\},\left\{\zeta_{j}(t)\right\}$ satisfy the algebraic constraint

$$
\rho(t)^{2} \prod_{k=1}^{N} \eta_{k}(t)=\prod_{k=1}^{N} \zeta_{k}(t) .
$$

Note that, as is easily established from the properties $(54)$ of $P(\zeta ; \rho)$, any function $z(\zeta, t)$ of the form (55) satisfies the following functional equation:

$$
z\left(\rho^{2} \zeta, t\right)=z(\zeta, t)
$$

Note also that $z(\zeta, t)$ is meromorphic everywhere in the plane except at $\zeta=0, \infty$. Such functions are known as loxodromic functions [21] and have recently been used in various contexts to solve a number of physically-distinct free boundary problems [14], [5], [7]. Richardson [14] provides a convenient and useful summary of the important properties of such functions based on a presentation given in [21]. The important point is that for domains $D(t)$ described by conformal maps $z(\zeta, t)$ that are loxodromic functions, $\mathcal{L}[h(z, t) ; D(t)]$ is a point differential functional of finite order. To see this, note that by Green's Theorem,

$$
\begin{aligned}
\mathcal{L}[h(z, t) ; D(t)]= & \frac{1}{2 i} \oint_{|\zeta|=1} h(z(\zeta, t), t) \bar{z}\left(\zeta^{-1}, t\right) z_{\zeta}(\zeta, t) d \zeta \\
& -\frac{1}{2 i} \oint_{|\zeta|=\rho} h(z(\zeta, t), t) \bar{z}\left(\rho^{2} \zeta^{-1}, t\right) z_{\zeta}(\zeta, t) d \zeta .
\end{aligned}
$$


But, using (57), this becomes

$$
\mathcal{L}[h(z, t) ; D(t)]=\frac{1}{2 i} \oint_{C_{0}} h(z(\zeta, t), t) \bar{z}\left(\zeta^{-1}, t\right) z_{\zeta}(\zeta . t) d \zeta,
$$

where the integration around $C_{0}$ is taken as anticlockwise around $|\zeta|=1$ and clockwise around $|\zeta|=\rho$. But since $h(z(\zeta, t), t)$ and $z_{\zeta}(\zeta, t)$ are analytic everywhere in $C_{0}$, while $\bar{z}\left(\zeta^{-1}, t\right)$ is meromorphic in $C_{0}$ with a finite number of poles it is clear that, by the residue theorem, the integral is given by a sum of a (finite number) of residue contributions from the points $\bar{\zeta}_{k}(t)^{-1}, k=1,2, \ldots, N$. Equivalently, $\mathcal{L}[h(z, t) ; D(t)]$ can be written in the form

$$
\mathcal{L}[h(z, t) ; D(t)]=\sum_{n=1}^{N} \sum_{k=0}^{n_{k}} a_{n k}(t) h^{(k)}\left(z_{n}(t), t\right),
$$

where $z_{n}(t)=z\left(\bar{\zeta}_{n}(t)^{-1}, t\right)$, i.e., $\mathcal{L}[h(z, t) ; D(t)]$ is a point differential functional of finite order.

The experiments of [3], [4] are concerned with initial configurations of fluid in which the two fluid interfaces are concentric circles forming a simple annulus $\rho(0)<r<R(0)$, say. It is easy to show that the model problem considered here admits a geometrically trivial solution in which the fluid domain remains a concentric annulus for all future times and where the outer interface is given by $r=R(t)$ and the inner interface is given by $r=\rho(t)$. The velocities of the two interfaces are given by

$$
\begin{aligned}
\dot{R}(t) & =\left(\frac{A}{2}\left(R^{2}-\rho^{2}\right)-B_{2}(t)\right) \frac{1}{R \log \frac{R}{\rho}}, \\
\dot{\rho}(t) & =\left(\frac{A}{2}\left(R^{2}-\rho^{2}\right)-B_{2}(t)\right) \frac{1}{\rho \log \frac{R}{\rho}} .
\end{aligned}
$$

This solution is essentially written down in [4] (although additional effects such as surface tension are also incorporated) and its linear stability is studied. Note that in these formulae, the dependence of the solution on the imposed pressure $B_{2}(t)$ inside the enclosed air bubble is directly apparent. In particular, note that the choice $B_{2}(t)=0$ in these formulae implies that both $\rho(t)$ and $R(t)$ increase (so that the fluid is flung out radially, as expected).

It is to be emphasized that the geometrically trivial solution (61) does not fall within the class of new exact solutions just derived. However, Gustafsson [12] has established that the set of doubly-connected quadrature domains is dense in the set of all doublyconnected domains. It must therefore be possible, given an initial domain that is a concentric annulus, to find an initial quadrature domain that is arbitrarily close to this concentric annulus. Such a domain will have a conformal map from a canonical parametric annulus $\rho_{0}<|\zeta|<1$ which is a loxodromic function. In general, it is to be expected that this loxodromic function might be of very large (but finite) order (i.e., might have to possess a large number of poles and zeros) if it is to map to the given concentric annulus to within a certain prespecified degree of accuracy. By the results obtained earlier in this paper, under evolution this initial domain will continue to be a quadrature domain and continue to have a corresponding loxodromic function conformal map. 
Consider a loxodromic function given by

$$
z(\zeta, 0)=R_{0} \zeta \frac{\prod_{j=1}^{N} Q_{N}\left(\frac{\zeta \rho_{0}^{2 / N}}{p_{0}} ; \rho_{0}\right)}{\prod_{j=1}^{N} Q_{N}\left(\frac{\zeta}{p_{0}} ; \rho_{0}\right)}
$$

where the function $Q_{N}(\zeta ; \rho)$ is defined by the infinite product expansion

$$
Q_{N}(\zeta ; \rho)=\left(1-\zeta^{N}\right) \prod_{k=1}^{\infty}\left(1-\rho^{2 k N} \zeta^{N}\right)\left(1-\rho^{2 k N} \zeta^{-N}\right)
$$

where $p_{0}$ is some real parameter such that $1<p_{0}<\rho_{0}^{-1}$. The real parameter $\rho_{0}$ is chosen so that $0<\rho_{0}<1$, and $R_{0}$ is taken to be real. This conformal map is of the form (55) but has been chosen in such a way that it has $N$ poles placed at points

$$
\zeta_{j}=p_{0} \omega_{N}^{j}, \quad j=0, \ldots, N-1,
$$

and $N$ zeros at points

$$
\eta_{j}=p_{0} \rho^{-2 / N} \omega_{N}^{j}, \quad j=0, \ldots, N-1,
$$

where $\omega_{N}=e^{2 \pi i / N}$ is the $N$ th root of unit. For $R_{0}=1$ and for suitably chosen $p_{0}$, a plot of the image of $\rho<|\zeta|<1$ under the mapping (62) for any $0<\rho<1$ shows it to be visually indistinguishable from the annulus $\rho<|z|<1$ in the limit as $N$ gets very large. Indeed, this can be shown analytically using the fact that $N \rightarrow \infty$. The details are omitted here.

In order to perform some concrete calculations of the exact solutions, it is necessary to specify some physical condition inside the enclosed central air bubble. In the physical experiments [3], [4], the hole through which the original blob of fluid is injected into the Hele-Shaw cell is contained within this air bubble and is left open to the atmosphere throughout the experiments. This makes the pressure inside the enclosed air bubble constant and effectively equal to that exterior to the fluid annulus (assuming the sides of the cell are also open to the atmosphere). The effect is that, as the cell starts to rotate, the pressure of the enclosed air remains constant and is unable to counteract the centrifugal forces, which causes the fluid in the annulus to be flung out radially and, as a direct result, the area of the enclosed air bubble to increase.

To accurately model this physical situation of the experiment it is necessary, strictly speaking, to solve the model mathematical problem using the condition that $B_{2}(t)=$ constant. This can indeed be done and, in the appendix, full details of the required equations are given. To summarize the results of the appendix, because the pressure is related to the field variable $\phi$, it is necessary at each instant to solve the full Dirichlet problem in the fluid annulus for $\phi$ and then impose the pressure condition at the inner interface. However, for the sake of purely illustrative calculations, we choose not to carry 
out this program in full, but settle for a reasonable approximation of the dynamics. As just discussed, the condition of constant pressure inside the enclosed air bubble results in the area of the enclosed bubble increasing. This rate of increase will depend upon the rate of rotation (which determines the size of the centrifugal force) and the imposed pressure $B_{2}(t)$ inside the enclosed air bubble. This is seen explicitly in the concentric annulus solution (61) and also in the appendix. In the following calculations, we model this situation in an approximate way by fixing the rate of rotation and simply choosing to specify that the area of the enclosed bubble increases at some externally specified rate. Since the area of the enclosed bubble for the near-concentric ammulus solutions described by the loxodromic conformal maps (62) is given, to very good accuracy, by the simple formula $\pi \rho^{2}$, the condition that the enclosed area increases is effectively equivalent to specifying that $\rho$ increases at some externally specified rate. Moreover, in our present calculations, the shapes of the annuli up to the point of breakdown remain reasonably close to concentric annuli, so that this specification on $\rho$ does indeed correspond to a general specification that the enclosed area increases.

We therefore choose some positive parameter $\sigma$ and choose to specify that

$$
\rho(t)=\rho_{0} e^{\sigma t}
$$

In this way, we effectively control the rate of increase of the enclosed bubble area. We now also choose $A(t)=1$, corresponding to stcady rotation. From earlier results, under the dynamics of the problem, the initial map (62) will evolve as

$$
z(\zeta, t)=R(t) \zeta \frac{\prod_{j=1}^{N} Q_{N}\left(\frac{\zeta \rho(t)^{2 / N}}{p(t)} ; \rho(t)\right)}{\prod_{j=1}^{N} Q_{N}\left(\frac{\zeta}{p(t)} ; \rho(t)\right)} .
$$

This map depends upon just three parameters $R(t), p(t)$, and $\rho(t) . \rho(t)$ is given by (66), which leaves us in need of two further equations. These two equations are, however, the exact analogues of (52) for the simply-comnected case and are given by

$$
\begin{gathered}
z_{1}(t)=z_{1}(0) e^{A t}=z\left(p(t)^{-1}, t\right), \\
a_{1}(t)=a_{1}(0)=\pi \operatorname{Residue}\left[\bar{z}\left(\zeta^{-1}, t\right) z_{\zeta}(\zeta, t) ; p(t)^{-1}\right] .
\end{gathered}
$$

To calculate solutions, at each time $t, \rho(t)$ is determined explicitly using (66). Equation (69) can then be used to provide an explicit formula for $R(t)$ in terms of the known $\rho(t)$ and the (as yet) unknown $p(t)$. Finally, (68) is solved, using Newton's method, to find the corresponding $p(t)$. Note that no integration in time is being performed; rather, the equations (66), (68), and (69) are purely algebraic in $R(t), \rho(t)$, and $p(t)$ for any given $t$. The equations are solved for the choice of the initial conditions

$$
R_{0}=1.0 . \quad \rho_{0}=0.4, \quad p_{0}=2.0
$$


with the fixed choice $A=1$ and with $N=51$. This $N$ is sufficiently large that the image of the parametric annulus $0.4<|\zeta|<1$ under the mapping (62) is visually indistinguishable from the annulus $0.4<|z|<1$. In the calculations, various different choices of the parameter $\sigma$ are chosen in order to examine the effects of different growth rates of the area of the enclosed bubble. Results for the values $\sigma=0.665,0.67$, and 0.675 are shown in Figures 1-6. As a check on the calculations, at certain times, the area of the fluid blob is explicitly calculated (by numerical integration using the conformal map) in order to ensure that it remains constant.

By plotting the image of the conformal map (67) for various values of the parameters, it is found that the map can lose univalency by means of cusp formation on either the inner or outer interface. Moreover, for odd values of $N$, and real values of the parameters $p$ and $R$, these cusps form on the negative real axis. This explains why we have chosen $N=51$-it is sufficiently large that the initial domain is indistinguishably close to the annulus $0.4<|z|<1.0$ and, because $N$ is odd, we can monitor breakdown of the solutions under evolution by tracking the two real quantities $z_{\zeta}(-1, t)$ and $z_{\zeta}(-\rho(t), t)$. If these two quantities hit zero, breakdown of the solution occurs via cusp formation on the outer and inner interfaces respectively.
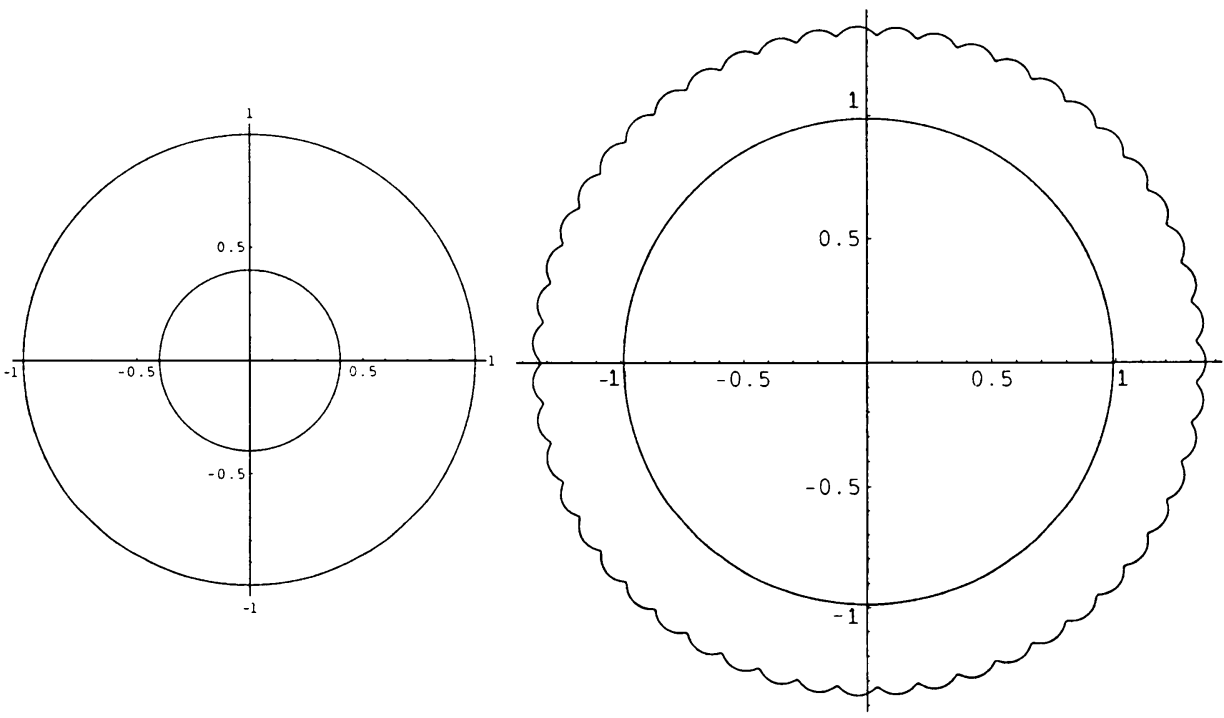

Fig. 1. $\sigma=0.665$; fluid annulus at times $t=0$ and $t=0.9175$

For the choice $\sigma=0.665$, Fig. 1 shows the initial fluid annulus and the annulus at $t=0.9175$ just before the solution breaks down by cusp formations on the outer interface. Figure 2 shows graphs of the quantities $z_{\zeta}(-1, t)$ and $z_{\zeta}(-\rho(t), t)$ and shows a rapid decrease in $z_{\zeta}(-1, t)$ towards zero. This corresponds to cusp formation on the outer interface on the negative real axis. Of course, by the rotational symmetry of the solution, cusps will form at 50 other symmetrically-disposed points around the outer interface. 


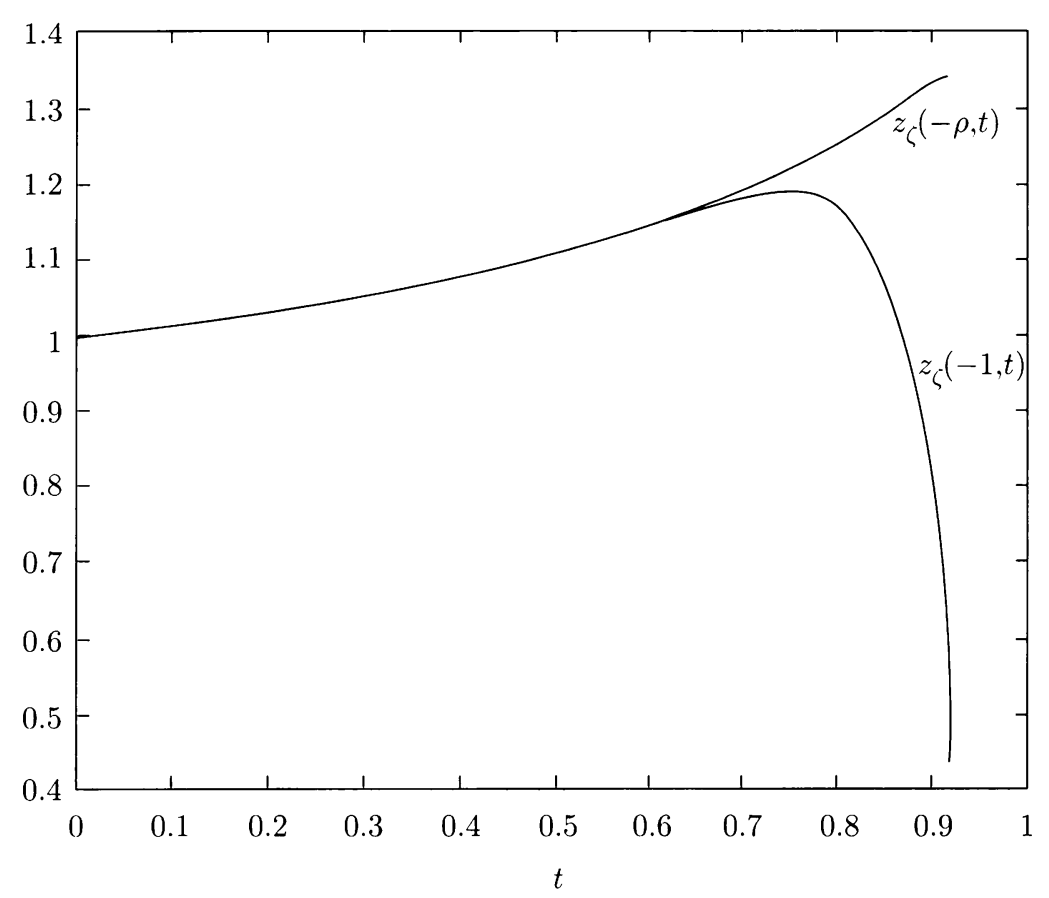

FIG. 2. Superposed graphs of $z_{\zeta}(-1, t)$ and $z_{\zeta}(-\rho(t), t)(\sigma=0.665)$.

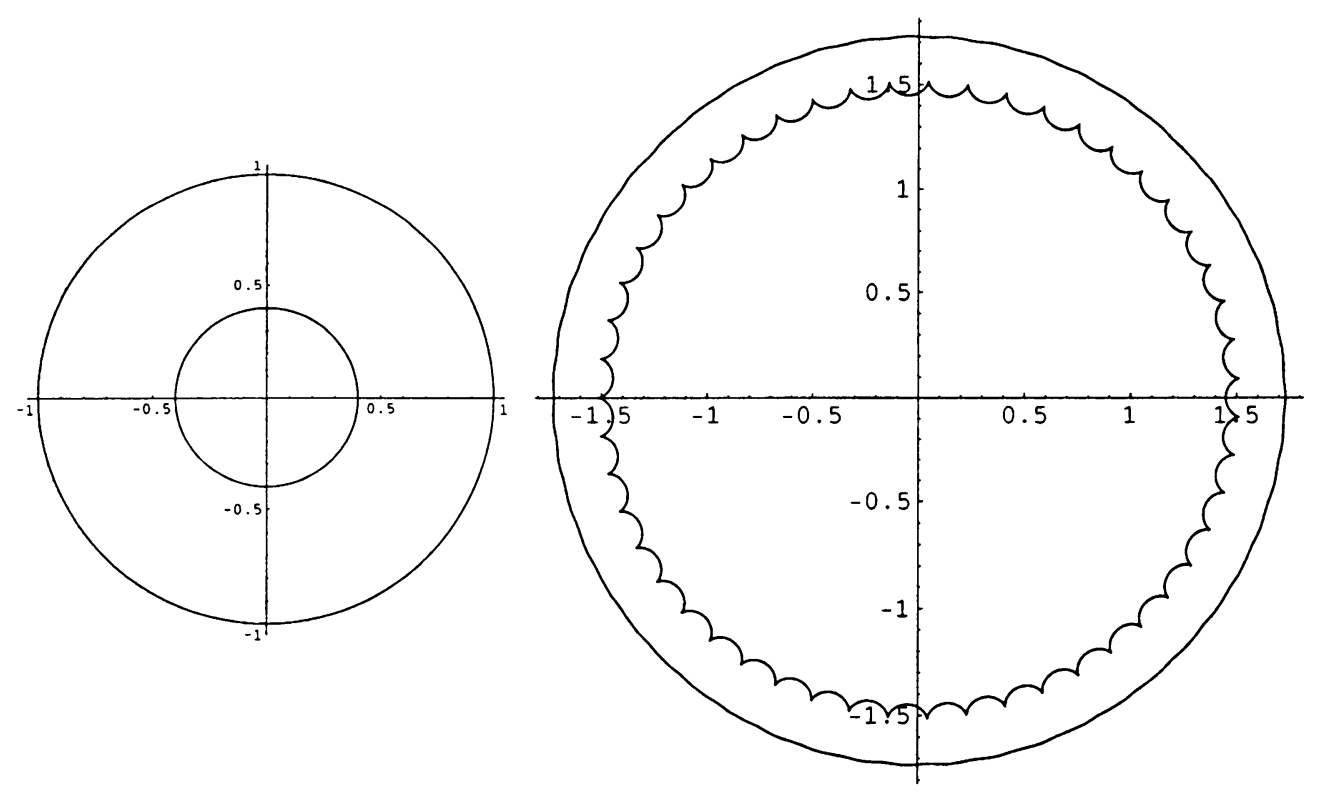

FIG. 3. $\sigma=0.67$; fluid annulus at times $t=0$ and $t=1.1405$ 


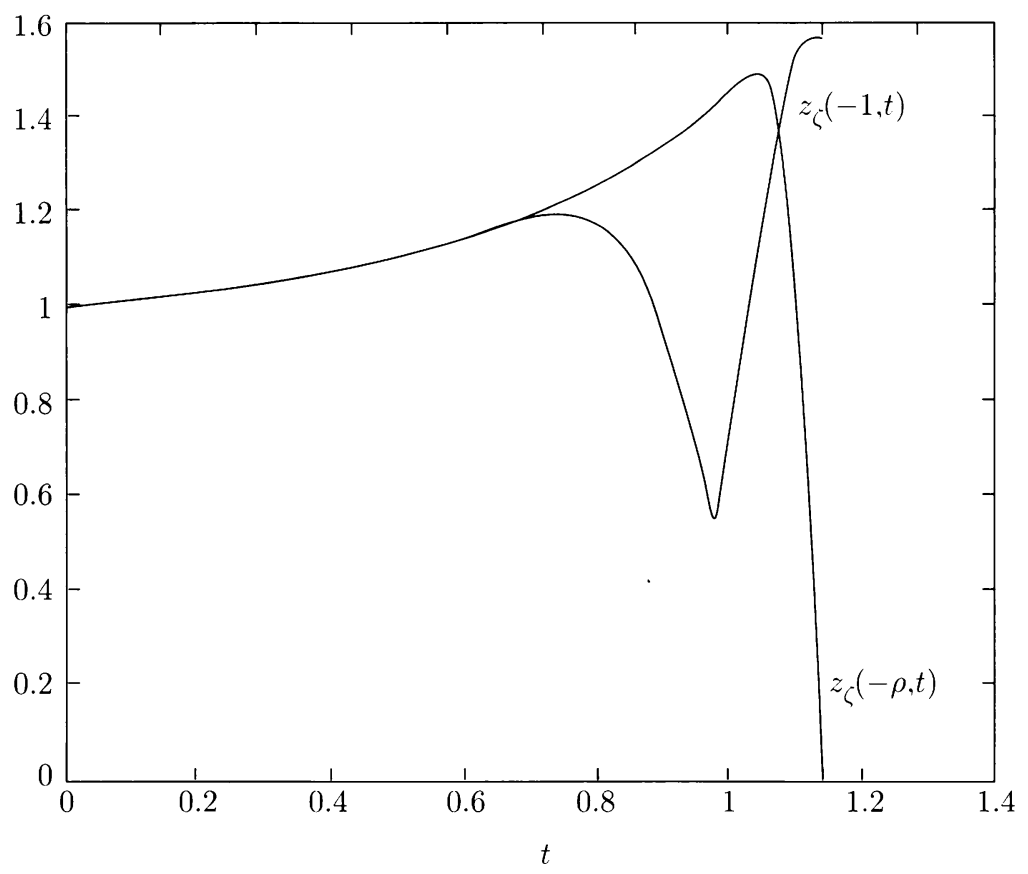

FIG. 4. Superposed graphs of $z_{\zeta}(-1, t)$ and $z_{\zeta}(-\rho(t), t)(\sigma=0.67)$

The choice $\sigma=0.67$ represents a value that is close to the critical value when there is a change-over in the nature of breakdown of the solution. In Fig. 3, the initial annulus and the annulus just before breakdown at $t=1.1405$ are shown. This time, breakdown occurs due to cusp formation on the inner interface. Figure 4 shows graphs of $z_{\zeta}(-1, t)$ and $z_{\zeta}(-\rho(t), t)$ and reveals particularly interesting behaviour; the graph of $z_{\zeta}(-1, t)$ shows a marked dip at $t \approx 0.98$, i.e., at around the time of breakdown of the solution (by cusp formation on the outer interface) in the previous case $\sigma=0.665$. Since the current $\sigma$-value is only 0.005 larger than this previous $\sigma$-value, one might have supposed that breakdown would indeed occur in the present case by means of the same mechanism as before and at around the same time. However, it is seen that the curve of $z_{\zeta}(-1, t)$ rapidly turns around before reaching zero (which would correspond to cusp formation in the outer interface) and the solution continues to exist until just after $t=1.1405$ when breakdown of the solution eventually occurs via cusp formation on the inner interface, as evinced by the fact that in Fig. $4, z_{\zeta}(-\rho(t), t)$ has now tended to zero. Also noteworthy is that the annulus has become very much thinner at the time of breakdown than in the previous example. It is remarkable that, although the values of $\sigma$ between this case and the case studied previously differ by just $\mathcal{O}\left(10^{-3}\right)$, the latter solution exists for approximately $\mathcal{O}\left(10^{-1}\right)$ additional units of time.

Finally, in Figs. 5 and 6 , we explore the case $\sigma=0.675$ in which the rate of increase of area of the enclosed air bubble is even greater. As expected, breakdown again occurs via cusp formation on the inner interface because the dominant instability turns out to be of the Saffman-Taylor type and, while we again see the dip in the value of $z_{\zeta}(-1, t)$, 
it is clear that the greater rate of increase of area of the enclosed bubble more effectively quashes the onset of the centrifugally-induced instability in the outer interface and the dip in $z_{\zeta}(-1, t)$ is less severe. Note also that the time of eventual breakdown is earlier than in the case $\sigma=0.67$ as might be expected since $\sigma=0.67$ is closer to the critical "cross-over" value at which the Saffman-Taylor type instability at the inner interface becomes important and it therefore takes longer for this instability to overcome the centrifugally-induced instability in the outer interface.
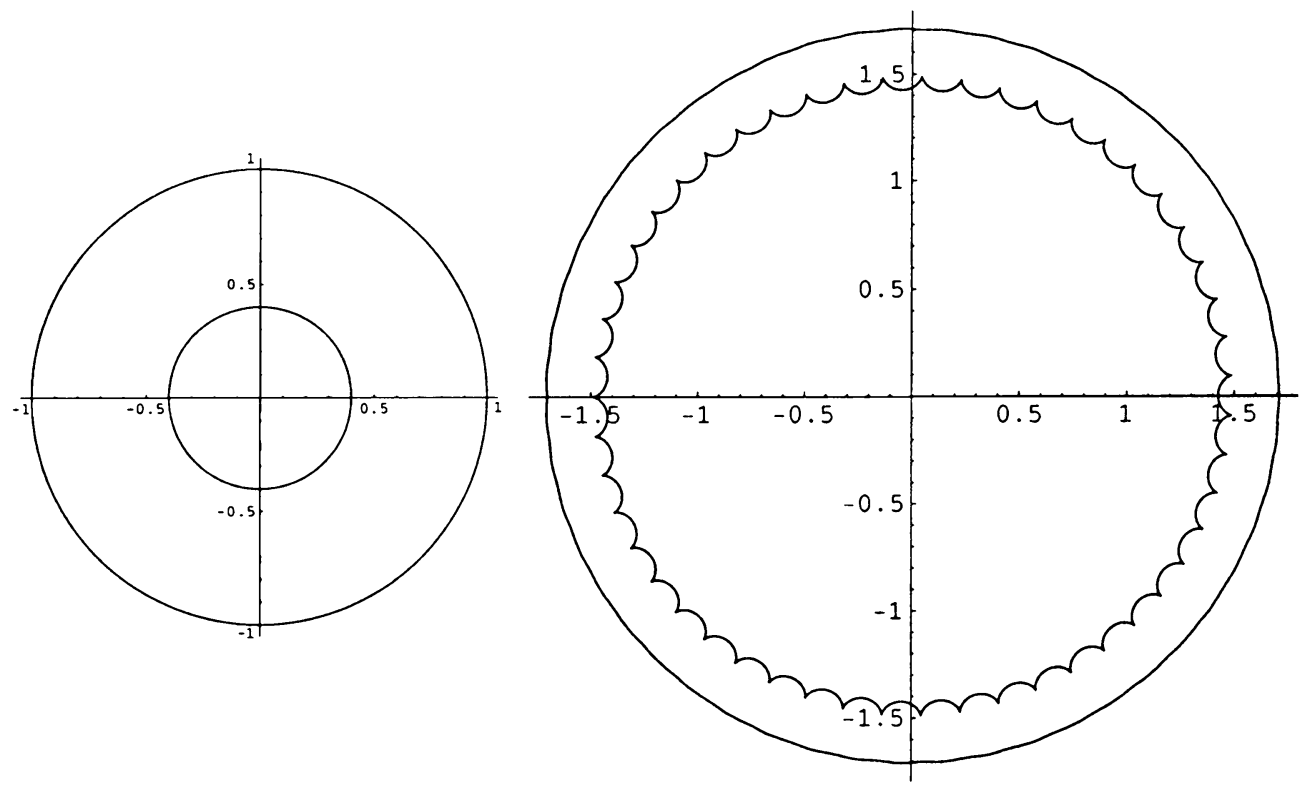

FIG. 5. $\sigma=0.675$; fluid annulus at times $t=0$ and $t=1.125$

4.3. Ill-posedness. Some important observations should be made. First, it is clear from the calculations that the mathematical model considered here is ill-posed. To see this, note that the initial conformal map (67) with initial parameters (70) gives an initial annulus which is indistinguishably close to the concentric annulus $0.4<|z|<1$ that would evolve according to the solution (61). However, while the solution (61) exists for all times (until the annulus becomes infinitely thin), the solution (67) breaks down in finite time (of order 1). Another manifestation of the nature of this ill-posedness can be demonstrated. If, instead of the initial conditions (70), the following initial conditions are used:

$$
R_{0}=1.0, \quad \rho_{0}=0.4, \quad p_{0}=1.5
$$

(note that $p_{0}$ has been decreased by 0.5 ) it is seen, by plotting the initial conformal map, that the image is again indistinguishably close to the concentric annulus $0.4<|z|<1.0$. However, when the evolution of this initial condition is calculated for the value $\sigma=0.665$, it is found that breakdown occurs by means of cusp formations on the outer interface at $t \approx 0.39$-i.e., significantly less than the critical breakdown time $t \approx 0.92$ for the set of 


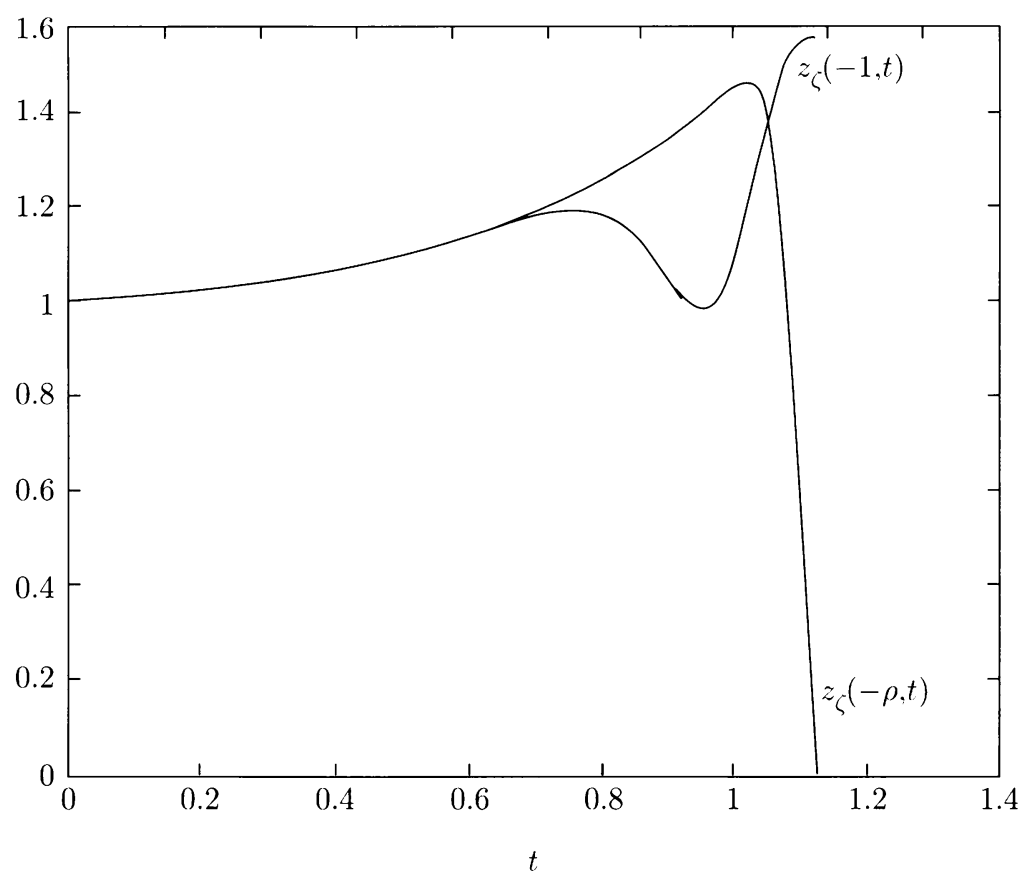

FIG. 6. Superposed graphs of $z_{\zeta}(-1, t)$ and $z_{\zeta}(-\rho(t), t)(\sigma=0.675)$

initial conditions (70). In this way, the nature of the ill-posedness is clearly seen-it is possible to choose initial conformal mapping parameters such that the initial images are indistinguishable, but which have markedly different nonlinear evolutions.

In spite of this ill-posedness, it is clear that this simple model displays all the qualitative mechanisms observed in the experiments. In particular, the competition between the centrifugally-induced instability mechanism and the Saffman-Taylor type instability is seen explicitly in the calculations. These two facts suggest that, with appropriate regularization (such as surface tension, contact angle effects, three-dimensional effects and/or wetting effects), the mathematical model stands a good chance of capturing the essential physics, while the existence of a dense class of exact solutions to the unregularized problem can only aid any analysis of a perturbatively regularized problem. We also point out that the present results constitute an essentially nonlinear analysis; a purely linear theory would not have been able to capture the "dip" behaviour seen in $z_{\zeta}(-1, t)$ in the cases $\sigma=0.67,0.675$ and a linear stability analysis would, at best, have led to the conclusion that breakdown of these solutions would occur by destabilization of the outer interface.

5. Discussion and summary. This paper has presented the new result that the problem of the evolution of a fluid annulus in a rotating Hele-Shaw cell admits exact, time-dependent solutions for the conformal mapping function having the general form 
given in (55) for any integer $N \geq 2$. The evolution equations for the finite set of parameters appearing in (55) have been explicitly derived. Illustrative solutions capturing the essential physical mechanisms have been calculated.

The initial value problem considered here has been seen, in an explicit way, to be ill-posed. The model predicts breakdown via cusp formation on the interfaces. As in the more traditional Hele-Shaw problem, in practice, this does not occur and the effects of small regularization effects (such as surface tension) can play unexpected roles in changing the predicted behaviour of the unregularized model [20]. Clearly, in this problem too, appropriate regularization of the model is needed before it can be expected to accurately reproduce physically-observable phenomena. Indeed, it has been shown in recent years (see Tanveer [20]) that it is very dangerous to assert any physical meaning to solutions of mathematically ill-posed problems of this kind.

For this reason, the solutions here are important. As in other types of Hele-Shaw free boundary problems, it is significant that the unregularized model admits exact solutions. In other types of Hele-Shaw problems, the existence of exact solutions to the unregularized model has proved to be of enormous value in facilitating further analyses of the regularized problem. When such regularization is introduced perturbatively, the exact zero-th order solutions can often simplify the pertubation analysis. For example, the evolution equations for the "daughter singularities" [19], [20] of Tanveer's asymptotic theory for regularizing the standard Hele-Shaw problem by means of very small surface tension, are ordinary differential equations whose coefficients are functions of the solution of the unregularized problem. That these solutions are known exactly in the standard HeleShaw model (with no rotation) represents a significant simplification of the asymptotic analysis. Indeed, it is clear that the asymptotic theory of Tanveer (see [20] for a useful summary of this theory) will also be relevant to a study of the current problem when regularization by means of very small surface tensions is considered. In the solutions presented here, it is seen that choosing the integer $N$ effectively selects a length-scale for the instability on the two interfaces. In the unregularized model considered here, there is no means of fixing this length-scale and in practice, it would be determined by the regularization mechanism, although this scaling can often be determined in very surprising ways (e.g., by means of an impacting daughter singularity cluster, see [20]). Indeed, it would be of great interest to explore the evolution of daughter singularities in the present problem where there exist two competing physical mechanisms which might affect their evolution and determine whether they first impact the inner or outer interface. Moreover, an impacting daughter singularity cluster is likely to more readily explain the typical finger-like formations (as opposed to cusps) that are seen to develop on the interfaces in the experiments (see, for example, [18]). This interesting problem is left for the future, and the present results are expected to form a basis for any developments in this direction.

The free boundary problem considered here has many mathematical similarities with the free boundary problem of Stokes flow driven by surface tension-a problem that scems, at first sight, to be very different mathematically because, while the present problem is governed by a second-order Laplace equation, the Stokes flow problem is governed by a fourth-order biharmonic equation. As evidence for this, note that cxact 
loxodromic function solutions have also recently been found for a special class of problems involving a "sintering" fluid annulus [5]. It is remarkable that two problems that are so different both physically and mathematically have such similar properties with regard to their free boundary evolution. This matter has been discussed in greater detail elsewhere [8] using the mathematical notion of Cauchy transforms.

Finally, the fact that the rotating Hele-Shaw problem preserves quadrature domains implies the existence of exact solutions for fluid regions of any finite connectivity. The doubly-connected fluid regions presented here have been constructed by exploiting conformal mapping theory. The construction of quadrature domains of higher (finite) connectivity is a challenging mathematical problem. However, Crowdy [9] has recently made some advances in this general area with a view to providing a practical method of constructing such domains for application in problems of genuine physical interest, such as that considered here.

Acknowledgments. The author gratefully acknowledges partial financial support for this research from the National Science Foundation (Grant numbers DMS-9803167 and DMS-9803358).

Appendix. Equation for $\rho(t)$ in terms of $B_{2}(t)$. In the experiments of [3], [4], the hole at the centre of the Hele-Shaw cell through which the fluid is injected is left open to the atmosphere. The effect is that the pressure of the air in the enclosed bubble is constant and equal to the pressure exterior to the fluid annulus (which has here, without loss of generality, been taken to be zero). Mathematically, the imposed condition is equivalent to specifying that $B_{2}(t)=0$. This physical requirement implicitly determines the evolution of the conformal modulus $\rho(t)$ as we will now illustrate in this appendix. It does this in a non-local way and, for the sake of completeness (and for those readers interested in solving the full equations of motion in this case) we now present the relevant equations.

Consider the conformal map $z(\zeta, t)$ from the annulus $C_{0}$ to the doubly-connected fluid domain $D(t)$ at each instant of time. It is required to find a function $\phi$ harmonic in the annulus $C_{0}$. As mentioned before, if a domain $D(t)$ is not simply-connected, there need not be an analytic function in $D(t)$ having $\phi$ as its real part. However, it is well known (see p. 455 of [16]) that in the case of an annulus, it is possible to overcome this difficulty and it is known that $\phi$, harmonic in $C_{0}$, can be written as

$$
\phi=\operatorname{Re}[\widetilde{G}(\zeta, t)]
$$

where

$$
\widetilde{G}(\zeta, t)=C(t) \log \zeta+H(\zeta, t)
$$

where $H(\zeta, t)$ is analytic and single-valued in $C_{0}$.

It is easily shown that on $|\zeta|=1$,

$$
z_{s}=\frac{i \zeta z_{\zeta}}{\left|z_{\zeta}\right|}
$$


while on $|\zeta|=\rho$,

$$
z_{s}=-\frac{i \zeta z_{\zeta}}{\rho\left|z_{\zeta}\right|}
$$

Using these expressions in the kinematic condition (13) provides that on $|\zeta|=1$,

$$
\operatorname{Re}\left[\frac{z_{t}}{\zeta z_{\zeta}}\right]=\operatorname{Re}\left[\frac{2 \phi_{\bar{z}}}{\zeta z_{\zeta}}\right]
$$

while on $|\zeta|=\rho$,

$$
\operatorname{Re}\left[\frac{z_{t}}{\zeta z_{\zeta}}\right]=\operatorname{Re}\left[\frac{2 \phi_{\bar{z}}}{\zeta z_{\zeta}}\right]-\frac{\dot{\rho}}{\rho}
$$

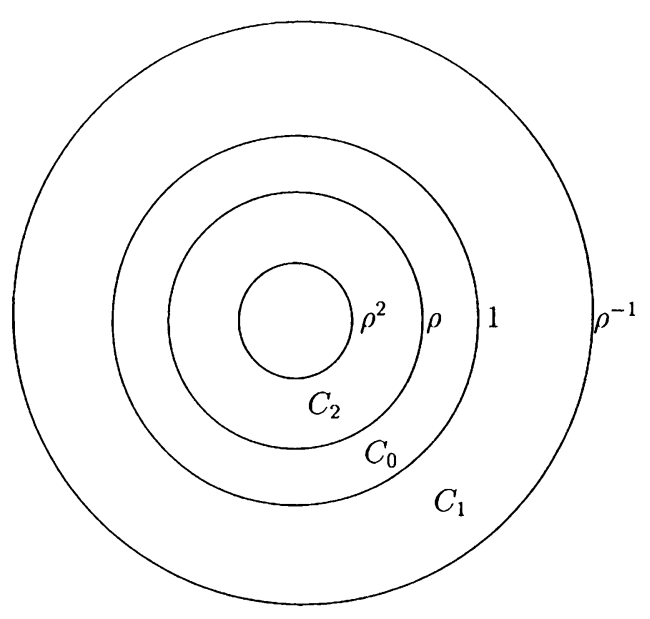

FIc: 7. Definition of annuli

Note that the quantity in square brackets on the left-hand side of (76) and (77) is known to be analytic and single-valued everywhere in $C_{0}$. It can therefore be written as the real part of a function analytic in $C_{0}$. Now define the function $G(\zeta, t)$ as follows:

$$
G(\zeta, t) \equiv 2 \phi_{z}=\frac{\tilde{G}_{\zeta}(\zeta, t)}{z_{\zeta}(\zeta, t)}
$$

Then Poisson's integral formula for an amnulus (see, e.g., [1]) implies that, for $\zeta \in C_{0}$,

$$
z_{t}(\zeta, t)=\zeta I(\zeta, t) z_{\zeta}(\zeta, t)
$$

where

$$
I(\zeta, t)=I^{+}(\zeta, t)-I^{-}(\zeta, t)+E_{1}(t)+i E_{2}(t)
$$

where

$$
I^{+}(\zeta, t)=\frac{1}{4 \pi i} \oint_{\left|\zeta^{\prime}\right|=1} \frac{d \zeta^{\prime}}{\zeta^{\prime}}\left(1-2 \frac{\zeta}{\zeta^{\prime}} \frac{P^{\prime}\left(\frac{\zeta}{\zeta^{\prime}}\right)}{P\left(\frac{\zeta}{\zeta^{\prime}}\right)}\right)\left[\frac{\bar{G}\left(\zeta^{\prime-1}, t\right)}{\zeta^{\prime} z_{\zeta}}+\frac{G\left(\zeta^{\prime}, t\right)}{\zeta^{\prime-1} \bar{z}_{\zeta}}\right)
$$


and

$$
I^{-}(\zeta, t)=\frac{1}{4 \pi i} \oint_{\left|\zeta^{\prime}\right|=\rho} \frac{d \zeta^{\prime}}{\zeta^{\prime}}\left(1-2 \frac{\zeta}{\zeta^{\prime}} \frac{P^{\prime}\left(\frac{\zeta}{\zeta^{\prime}}\right)}{P\left(\frac{\zeta}{\zeta^{\prime}}\right)}\right)\left[\frac{\bar{G}\left(\rho^{2} \zeta^{\prime-1}, t\right)}{\zeta^{\prime} z_{\zeta}}+\frac{G\left(\zeta^{\prime}, t\right)}{\rho^{2} \zeta^{\prime-1} \bar{z}_{\zeta}}-\frac{2 \dot{\rho}}{\rho}\right],
$$

where $E_{1}(t)$ is a real function of time given by

$$
E_{1}(t)=-\frac{1}{4 \pi i} \oint_{\left|\zeta^{\prime}\right|=\rho} \frac{d \zeta^{\prime}}{\zeta^{\prime}}\left[\frac{\bar{G}\left(\rho^{2} \zeta^{\prime-1}, t\right)}{\zeta^{\prime} z_{\zeta}}+\frac{G\left(\zeta^{\prime}, t\right)}{\zeta^{\prime-1} \bar{z}_{\zeta}}-\frac{2 \dot{\rho}}{\rho}\right] .
$$

Using the rotational degree of freedom in the Riemann Mapping Theorem, we can set $E_{2}(t)=0$ without loss of generality. Note that it is a necessary condition (for $\frac{z_{t}}{\zeta z_{\zeta}}$ to be analytic and single-valued in $\left.C_{0}\right)$ that the average of $\operatorname{Re}\left[\frac{z_{t}}{\zeta z_{\zeta}}\right]$ over $|\zeta|=1$ be equal to its average over $|\zeta|=\rho$. This condition provides the evolution equation for $\rho(t)$. Specifically,

$$
\begin{aligned}
\dot{\rho}=\frac{\rho}{4 \pi i}\left(\oint_{\left|\zeta^{\prime}\right|=\rho} \frac{d \zeta^{\prime}}{\zeta^{\prime}}\right. & {\left[\frac{\bar{G}\left(\rho^{2} \zeta^{\prime-1}, t\right)}{\zeta^{\prime} z_{\zeta}}+\frac{G\left(\zeta^{\prime}, t\right)}{\rho^{2} \zeta^{\prime-1} \bar{z}_{\zeta}}\right] } \\
& \left.-\oint_{\left|\zeta^{\prime}\right|=1} \frac{d \zeta^{\prime}}{\zeta^{\prime}}\left[\frac{\bar{G}\left(\zeta^{\prime-1}, t\right)}{\zeta^{\prime} z_{\zeta}}+\frac{G\left(\zeta^{\prime}, t\right)}{\zeta^{\prime-1} \bar{z}_{\zeta}}\right]\right) .
\end{aligned}
$$

Using the given pressure boundary conditions on the two interfaces it can be shown that

$$
\frac{\bar{G}\left(\zeta^{-1}, t\right)}{\zeta z_{\zeta}(\zeta, t)}=\frac{G(\zeta, t)}{\zeta^{-1} \bar{z}_{\zeta}\left(\zeta^{-1}, t\right)}+A(t)\left(\frac{z(\zeta, t)}{\zeta z_{\zeta}(\zeta, t)}-\frac{\bar{z}\left(\zeta^{-1}, t\right)}{\zeta^{-1} \bar{z}_{\zeta}\left(\zeta^{-1}, t\right)}\right)
$$

on $|\zeta|=1$ and

$$
\frac{\bar{G}\left(\rho^{2} \zeta^{-1}, t\right)}{\zeta z_{\zeta}(\zeta, t)}=\frac{G(\zeta, t)}{\rho^{2} \zeta^{-1} \bar{z}_{\zeta}\left(\zeta^{-1}, t\right)}+A(t)\left(\frac{z(\zeta, t)}{\zeta z_{\zeta}(\zeta, t)}-\frac{\bar{z}\left(\rho^{2} \zeta^{-1}, t\right)}{\rho^{2} \zeta^{-1} \bar{z}_{\zeta}\left(\rho^{2} \zeta^{-1}, t\right)}\right)
$$

on $|\zeta|=\rho$. By analytic continuation, these relations hold off the circles $|\zeta|=1$ and $|\zeta|=\rho$. Substituting for the left-hand sides of (85) and (86) in the equation for $\dot{\rho}$, leads to

$$
\begin{aligned}
\dot{\rho}=\frac{\rho}{4 \pi i}\left(\oint_{\left|\zeta^{\prime}\right|=\rho} \frac{d \zeta^{\prime}}{\zeta^{\prime}}\right. & {\left[\frac{2 G\left(\zeta^{\prime}, t\right)}{\rho^{2} \zeta^{\prime-1} \bar{z}_{\zeta}\left(\rho^{2} \zeta^{\prime-1}, t\right)}+\frac{A z\left(\zeta^{\prime}, t\right)}{\zeta^{\prime} z_{\zeta}\left(\zeta^{\prime}, t\right)}-\frac{A \bar{z}\left(\rho^{2} \zeta^{\prime-1}, t\right)}{\rho^{2} \zeta^{\prime-1} \bar{z}_{\zeta}\left(\rho^{2} \zeta^{\prime-1}, t\right)}\right] } \\
& \left.-\oint_{\left|\zeta^{\prime}\right|=1} \frac{d \zeta^{\prime}}{\zeta^{\prime}}\left[\frac{2 G\left(\zeta^{\prime}, t\right)}{\zeta^{\prime-1} \bar{z}_{\zeta}\left(\zeta^{\prime-1}, t\right)}+\frac{A z\left(\zeta^{\prime}, t\right)}{\zeta^{\prime} z_{\zeta}\left(\zeta^{\prime}, t\right)}-\frac{A \bar{z}\left(\zeta^{\prime-1}, t\right)}{\zeta^{\prime-1} \bar{z}_{\zeta}\left(\zeta^{\prime-1}, t\right)}\right]\right) .
\end{aligned}
$$

Equation (87) represents the general equation of evolution for $\rho(t)$. However, if $z(\zeta, t)$ is known to be a loxodromic function, then $\zeta z_{\zeta}(\zeta, t)$ is also a loxodromic function. Then, using the fact that $\frac{z(\zeta, t)}{\zeta z_{\zeta}(\zeta, t)}$ is known to be analytic in $C_{0}$, the equation for $\dot{\rho}$ reduces to

$$
\dot{\rho}=-\frac{\rho}{4 \pi i} \oint_{C_{0}}\left[\frac{2 G\left(\zeta^{\prime}, t\right)-A(t) \bar{z}\left(\zeta^{\prime-1}, t\right)}{\bar{z}_{\zeta}\left(\zeta^{\prime-1}, t\right)}\right] d \zeta^{\prime} .
$$

It is interesting to point out that because $G(\zeta, t)$ is known to be analytic in $C_{0}$, the integrand in (88) is meromorphic in $C_{0}$ and so, in principle, the integral (88) can be reduced to a finite sum of residue contributions from the $2 N$ zeros of $\bar{z}_{\zeta}\left(\zeta^{-1}, t\right)$ inside $C_{0}$ (assuming $z(\zeta, t)$ is a loxodromic function of order $N$ ). 
To obtain an expression for $G(\zeta, t)$ valid inside $C_{0}$, note that from $(78)$ we have $G(\zeta, t)$ if we have an expression for $\widetilde{G}(\zeta, t)$ valid inside $C_{0}$. But

$$
\phi=\operatorname{Re}[\widetilde{G}(\zeta, t)]=\frac{A(t)}{2} z \bar{z}=B_{j}(t) \quad \text { on } \partial D_{j}(t)
$$

so that it is clear that $\widetilde{G}(\zeta, t)$ is the solution of the following Dirichlet problem in an annulus $C_{0}$ :

$$
\begin{aligned}
& \operatorname{Re}[\widetilde{G}(\zeta, t)]=\frac{A(t)}{2} z(\zeta, t) \bar{z}\left(\zeta^{-1}, t\right) \quad \text { on }|\zeta|=1, \\
& \operatorname{Re}[\widetilde{G}(\zeta, t)]=\frac{A(t)}{2} z(\zeta, t) \bar{z}\left(\rho^{2} \zeta^{-1}, t\right)+B_{2}(t), \quad \text { on }|\zeta|=\rho
\end{aligned}
$$

The solution to this Dirichlet problem can be written ([13], [16])

$$
\widetilde{G}(\zeta, t)=C(t) \log \zeta+H(\zeta, t)
$$

where $C(t)$ is a real function of time, and $H(\zeta, t)$ is analytic and single-valued in $C_{0}$ having the following boundary values:

$$
\begin{aligned}
& \operatorname{Re}[H(\zeta, t)]=\frac{A(t)}{2} z(\zeta, t) \bar{z}\left(\zeta^{-1}, t\right) \quad \text { on }|\zeta|=1, \\
& \operatorname{Re}[H(\zeta, t)]=\frac{A(t)}{2} z(\zeta, t) \bar{z}\left(\rho^{2} \zeta^{-1}, t\right)+B_{2}(t)-C(t) \log \rho(t) \quad \text { on }|\zeta|=\rho .
\end{aligned}
$$

Again using Poisson's integral formula for an annulus [1], it can be deduced that

$$
H(\zeta, t)=J^{+}(\zeta, t)-J^{-}(\zeta, t)+D_{1}(t)+i D_{2}(t)
$$

where

$$
J^{+}(\zeta, t)=\frac{1}{2 \pi i} \oint_{\left|\zeta^{\prime}\right|=1} \frac{d \zeta^{\prime}}{\zeta^{\prime}}\left(1-2 \frac{\zeta}{\zeta^{\prime}} \frac{P^{\prime}\left(\frac{\zeta}{\zeta^{\prime}}\right)}{P\left(\frac{\zeta}{\zeta^{\prime}}\right)}\right)\left[\frac{A(t)}{2} z(\zeta, t) \bar{z}\left(\zeta^{-1}, t\right)\right]
$$

and

$$
\begin{array}{r}
J^{-}(\zeta, t)=\frac{1}{2 \pi i} \oint_{\left|\zeta^{\prime}\right|=\rho} \frac{d \zeta^{\prime}}{\zeta^{\prime}}\left(1-2 \frac{\zeta}{\zeta^{\prime}} \frac{P^{\prime}\left(\frac{\zeta}{\zeta^{\prime}}\right)}{P\left(\frac{\zeta}{\zeta^{\prime}}\right)}\right)\left[\frac{A(t)}{2} z(\zeta, t) \bar{z}\left(\rho^{2} \zeta^{-1}, t\right)\right. \\
\left.+B_{2}(t)-C(t) \log \rho(t)\right]
\end{array}
$$

provided that

$$
\begin{array}{r}
\oint_{\left|\zeta^{\prime}\right|=1} \frac{d \zeta^{\prime}}{\zeta^{\prime}}\left[\frac{A(t)}{2} z\left(\zeta^{\prime}, t\right) \bar{z}\left(\zeta^{\prime-1}, t\right)\right]=\oint_{\left|\zeta^{\prime}\right|=\rho} \frac{d \zeta^{\prime}}{\zeta^{\prime}}\left[\frac{A(t)}{2} z\left(\zeta^{\prime}, t\right) \bar{z}\left(\rho^{2} \zeta^{\prime-1}, t\right)\right. \\
\left.+B_{2}(t)-C(t) \log \rho(t)\right]
\end{array}
$$

and where $D_{1}(t)$ and $D_{2}(t)$ are constants (which are unimportant in the present analysis). (96) is a necessary condition for the single-valuedness of $H(\zeta, t)$ in $C_{0}$. For the class of 
exact solutions where $z(\zeta, t)$ remains loxodromic, (96) provides the following equation for $C(t)$ in terms of the externally specified $B_{2}(t)$ :

$$
C(t)=\frac{1}{\log \rho(t)}\left[B_{2}(t)-\frac{A(t)}{4 \pi i} \oint_{C_{0}} \frac{d \zeta^{\prime}}{\zeta^{\prime}} z\left(\zeta^{\prime}, t\right) \bar{z}\left(\zeta^{\prime-1}, t\right)\right] .
$$

As a consistency check, note that this equation provides a real function $C(t)$ and hence guarantees that $\phi$ is single-valued in $C_{0}$.

To summarize the formulae, once $A(t)$ and $B_{2}(t)$ are specified, $C(t)$ can be deduced from (97). Given $C(t), J^{+}(t)$ and $J^{-}(t)$ can be computed from (94) and (95) so that $H_{\zeta}(\zeta, t)$ is known. $(91)$ implies that

$$
\widetilde{G}_{\zeta}(\zeta, t)=\frac{C(t)}{\zeta}+H_{\zeta}(\zeta, t)=\frac{C(t)}{\zeta}+J_{\zeta}^{+}(\zeta, t)-J_{\zeta}^{-}(\zeta, t)
$$

so that

$$
G(\zeta, t)=\frac{1}{z_{\zeta}(\zeta, t)}\left[\frac{C(t)}{\zeta}+J_{\zeta}^{+}(\zeta, t)-J_{\zeta}^{-}(\zeta, t)\right]
$$

which is required in Eq. (88) for $\dot{\rho}$.

It is clear that the dependence of $\rho(t)$ on the pressure $B_{2}(t)$ inside the enclosed bubble is a rather complicated one; however, if required, it can be computed in the way just described.

\section{REFERENCES}

[1] N. I. Akhiezer, Elements of the Theory of Elliptic Functions, Translations of Mathematical Monographs, No. 79, Amer. Math. Soc., Providence, RI, 1990

[2] L. Carrillo, F. X. Magdeleno, J. Casademunt, and J. Ortin, Experiments in a rotating Hele-Shaw cell, Phys. Rev. E 54, 6260 (1996)

[3] L. Carrillo, J. Soriano, and J. Ortin, Radial displacement of a fluid annulus in a rotating Hele-Shaw cell, Phys. Fluids 11 (4), 778 (1999)

[4] L. Carrillo, J. Soriano, and J. Ortin, Interfacial instabilities of a fluid annulus in a rotating HeleShaw cell, Phys. Fluids 12 (7), 1685-1698 (2000)

[5] D. Crowdy and S. Tanveer, A theory of exact solutions for annular viscous blobs, J. Nonlinear Sci. 8, 261-279 (1998)

[6] D. G. Crowdy, A note on viscous sintering and quadrature identities, European J. Appl. Math. 10, 623-634 (1999)

[7] D. G. Crowdy, Exact solutions for steady capillary waves on a fluid annulus, J. Nonlinear Sci. 9, 615-640 (1999)

[8] D. G. Crowdy, On a class of geometry-driven free boundary problems, SIAM J. Appl. Math. (to appear)

[9] D. G. Crowdy, On the construction of multiply-connected quadrature domains, SIAM J. Appl. Math. (to appear)

[10] P. J. Davis, The Schwarz Function and its Applications, Carus Mathematical Monographs, No. 17, The Mathematical Association of America, Buffalo, NY, 1974

[11] V. M. Entov, P. I. Etingof, and D. Ya. Kleinbock, On nonlinear interface dynamics in Hele-Shaw flows, European J. Appl. Math. 6, 399-420 (1995)

[12] B. Gustafsson, Quadrature domains and the Schottky double, Acta Appl. Math. 1, 209-240 (1983)

[13] M. Heins, Complex Function Theory, Academic Press, New York, 1968

[14] S. Richardson, Hele-Shaw flows with time-dependent free boundaries involving a concentric annulus, Philos. Trans. Roy. Soc. London Ser. A 354, 2513-2553 (1996)

[15] P. G. Saffman and G. I. Taylor, The penetration of a fluid into a porous medium of Hele-Shaw cell containing a more viscous fluid, Proc. Roy. Soc. London A, 245, 312-329 (1958) 
[16] S. Saks and A. Zygmund, Analytic Functions, Elsevier Publishing Company, Amsterdam, London, New York, 1971

[17] L. W. Schwartz, Instability and fingering in a rotating Hele-Shaw cell or porous medium, Phys. Fluids 1 (2), 167-169 (1989)

[18] M. Siegel, S. Tanveer, and W.-S. Dai, Singular effects of surface tension in evolving Hele-Shaw flows, J. Fluid Mech. 323, 201-236 (1996)

[19] S. Tanveer, Evolution of a Hele-Shaw interface for small surface tension, Philos. Trans. Roy. Soc. London A, 343, 155-204 (1993)

[20] S. Tanveer, Surprises in viscous fingering, J. Fluid Mech. 409, 273-308 (2000)

[21] G. Valiron, Cours d'Analyse Mathématique: Théorie des Fonctions, 2nd Edition, Masson et Cie, Paris, 1947 